\title{
Comparative Analysis of QoE Multipath TCP Congestion Control LIA, CUBIC, and WVEGAS on Video Streaming
}

\author{
Fiqqih M. Susanto ${ }^{1}$, Aji G. Putrada², Siti A. Karimah ${ }^{3}$ \\ ${ }^{1,2,3}$ School of Computing, Telkom University \\ Bandung, Indonesia \\ ${ }^{1}$ fiqqihmaulana@student.telkomuniversity.ac.id, 2ajigps@telkomuniversity.ac.id, \\ ${ }^{3}$ karimahsiti@telkomuniversity.ac.id
}

\begin{abstract}
Transmission Control Protocol (TCP) is a type of protocol that allows a collection of computers to communicate and exchange data within a network. Nowadays electronic devices such as tablets, personal computers and smartphones can use more than one network at the same time, but this is not supported by the characteristics of TCP which can only use one path on the network. To solve this condition there are several new generations of standardized network protocols. Multipath TCP is a development of TCP, Multipath which is a new generation network protocol that allows traffic to use multiple paths in the network. In addition to being able to use multiple paths on multipath TCP, there are several congestion control algorithms including LIA, CUBIC and WVEGAS congestion control algorithms. Tests conducted in this study were to compare the performance of congestion control LIA, CUBIC and WVEGAS to improve the quality of video streaming. From the test results, CUBIC is better than WVEGAS and LIA because the QoS and QoE video streaming test for CUBIC in all testing environments have better results than others.
\end{abstract}

Keywords: CUBIC, LIA, Multipath TCP, WVEGAS

\section{INTRODUCTION}

$\mathbf{N}$ OWADAYS electronic devices such as tablets, personal computers and smartphones can use more than one network at the same time, but this is not supported by the characteristics of TCP which can only use one path on the network. To solve this condition there are several new generations of standardized network protocols. Multipath TCP which is the development of TCP is a new generation network protocol that can help us to use multiple paths in network. By using Multipath TCP, devices can use more than one network and will be able to take advantage of the two or more available networks to get better quality of network [1][2].

Furthermore, many services will be improved by the presence of this multipath TCP technology, especially multimedia services such as video streaming [3]. This video streaming service has become one of the most used services on the Internet. Starting from entertainment to learning methods, especially in online learning models [4]. Therefore, improving the quality of services is needed. Since quality is often determined by the user, the 
quality from the user's point of view must be evaluated and this is done by the quality of experience (QoE) parameter [1].

In this research, Multipath TCP is implemented with congestion control LIA, CUBIC and WVEGAS to test the QoE (Quality of Experience) of Video Streaming. The LIA, CUBIC and WVEGAS congestion control algorithms were chosen because there have been studies that have shown a comparison of the performance of these algorithms against video streaming [5-7].

Based on reference [8-10], it is said that QoE is the overall acceptance of an application, which is perceived subjectively by end-users. Therefore, this research uses the Throughput parameter as an objective assessment of QoS, as well as image quality, sound quality and the length of video buffering taken from the end-user questionnaire, which will describe the quality from the subjective side in the form of QoE.

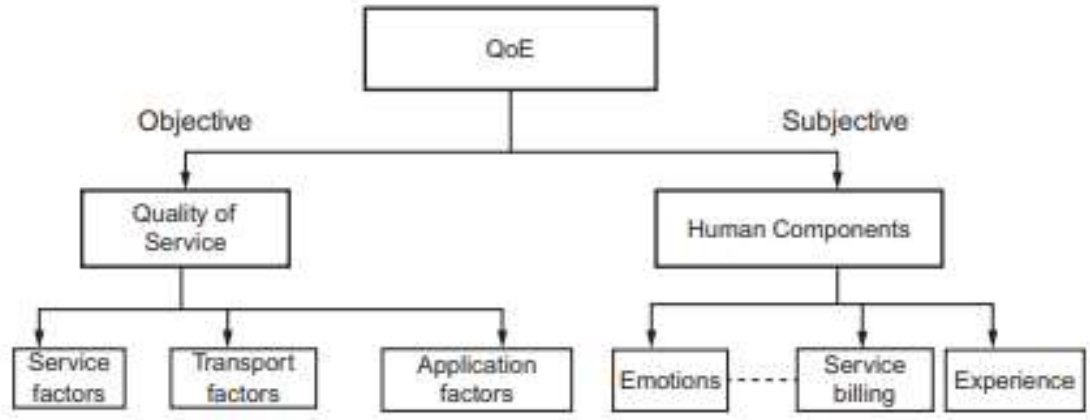

Figure 1. Factors Contributing to QoE [16]

\section{LITERATURE REVIEW}

In 2018, Jorge Mena, Yuanzhi Gao and Mario Gerla conducted a research entitled "MPTCP Path Selection using CapProbe". This research discusses the use of Multipath TCP technology to select the best network path [11]. This paper aims to measure and determine the best path on the network used by users, the method used by this paper is called packet pair algorithm. The algorithm used in this paper is successful in measuring and determining the best path and diverting the network path to the right path without user intervention.

In 2015, Yasuomi Muraki and Yoshihiro Ito conducted a research entitled "Study on Effect of Congestion Control of Multipath TCP on Web - QoE" [1]. This study discusses mobile devices that have many networks and can be used at one time, but the TCP protocol can only use one network path which causes network connection to be less than optimal, especially when searching the internet such as maps and online shopping sites. This paper aims to test QoE on map web services and online shopping sites based on the level of effectiveness, efficiency and QoS generated using the TCP Reno, TCP Vegas and MPTCP LIA congestion control and proven that congestion control affects QoS.

In 2015, Anilal P *, B V Sainandan and Siva Sankara Sai S conducted a study entitled "Experimentation and Analysis of Multipath TCP" which conducted tests on multipath aggregation, increased throughput, increased safety, and the use of congestion control [12]. Researchers experimented and analyzed the relationship between throughput and various congestion control with several topologies and scenarios. In this study, the authors used the latest version of the Linux MPTCP implementation developed by Universite Catholique deLouvain and proved that with network path aggregation it can increase throughput and from all experiments, the LIA algorithm shows better results than all the scenarios that have been carried out. 
In 2017, Mijanur Ruansa Palash and Kang Chen conducted a research entitled "Improved WiFi Network Performance Under MPTCP Users" [13]. This paper discusses the use of MPTCP on a WiFi network which can cause a decrease in network performance caused by a weak WiFi signal. So, this research applies the subflow prioritization method to maintain MPTCP performance by suppressing the influence of small signals that cause reduced throughput. The method adopted is proven to prevent reduction in throughput.

This research was conducted using two ethernet links that will represent MPTCP. In addition, we used three types of congestion control algorithms that are commonly used, namely Cubic, LIA and WVegas. Analyzes were carried out on the parameters of Quality of Service as well as Quality of Experience, to determine which congestion control algorithm has the best results.

\section{RESEARCH METHOD}

Figure 1 shows the flowchart of the research to understand the simulation design process. This research used 2 operating systems, Windows 7 and Linux Ubuntu 14.04. Multipath TCP is installed on Linux Ubuntu 14.04 along with its congestion control. LIA, CUBIC and WVEGAS were used as congestion control since they had been tested and are proven methods from previous research. The simulation starts with designing the topology to be used, then installing Linux, implementing multipath TCP, and setting congestion control on multipath TCP. The test is done several times to test the network with the video streaming service. The test is done by differentiating the environment and congestion control on multipath TCP. After successfully conducting the simulation, data collection, data analysis and conclusions will be drawn from the results that have been obtained. Congestion control wvegas works by providing a fair traffic shifting so that each flow will as much as possible balance the number of packets for each path by giving large cwnd for paths that have large traffic and small cwnd for paths that have small traffic, cubic congestion control works by enlarging or reduce the window size based on current network conditions and congestion control LIA works by equalizing the windows size and equalizing all available paths.

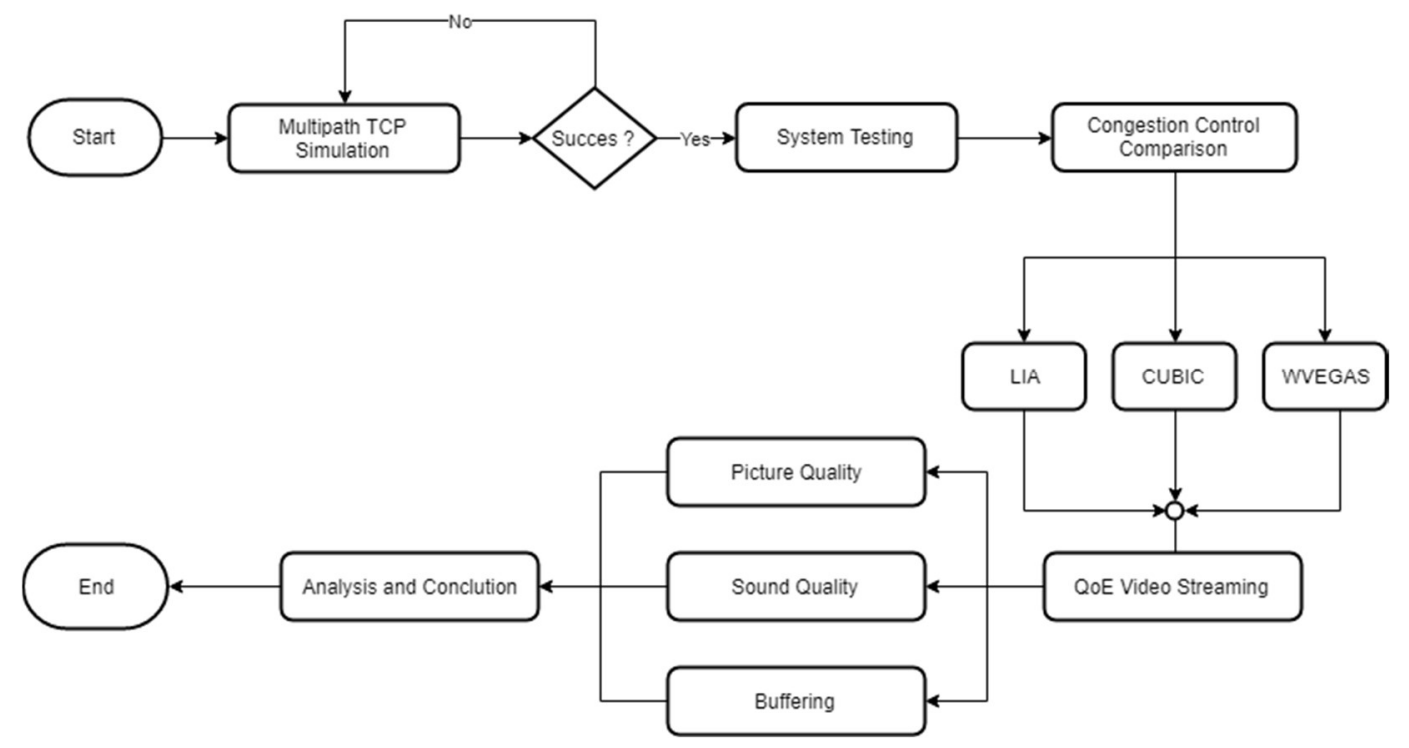

Figure 2. System Flowchart 


\section{A. System Topology}

In this research, Linux and Windows are used as two operating systems for simulations. Windows is used as a traffic generator while Linux functions as client and server. In this test, the server will do live streaming, then the client will stream the video played by the server. To manage network congestion, traffic generator is used to deliver traffic to the client network which will cause congestion to the client network, so we can test the congestion control that has been set and see the results obtained then compare the results. The network emulator is used to regulate packet loss on the specified path and determine how much bandwidth the line has.

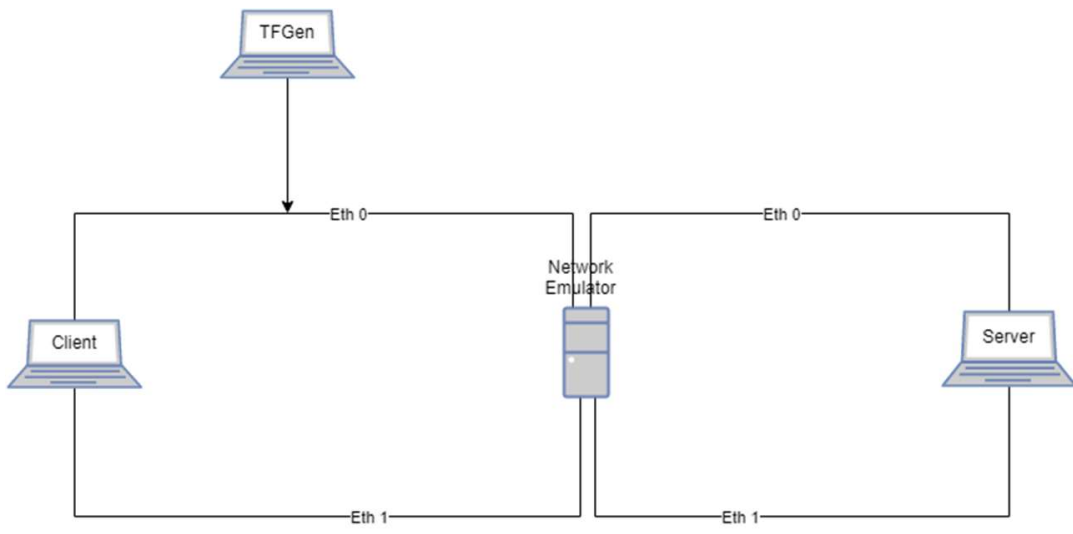

Figure 3. Simulation Topology

\section{B. Congestion Control Algorithms}

1) CUBIC: Cubic is the development of the BIC-TCP congestion control, cubic congestion control has a cwnd control mechanism based on the RTT time conditions at that time, cubic will increase the cwnd gradually until loss occurs, the cwnd value becomes the wmax value when the cwnd value reaches the maximum. Cubic determines the cwnnd value after loss when the window exceeds the wmax value [14].

2) LIA: LIA is an extension of the TCP-RENO congestion control, the LIA congestion control uses the linked increase method which works by increasing or decreasing the congestion window in a flow [15].

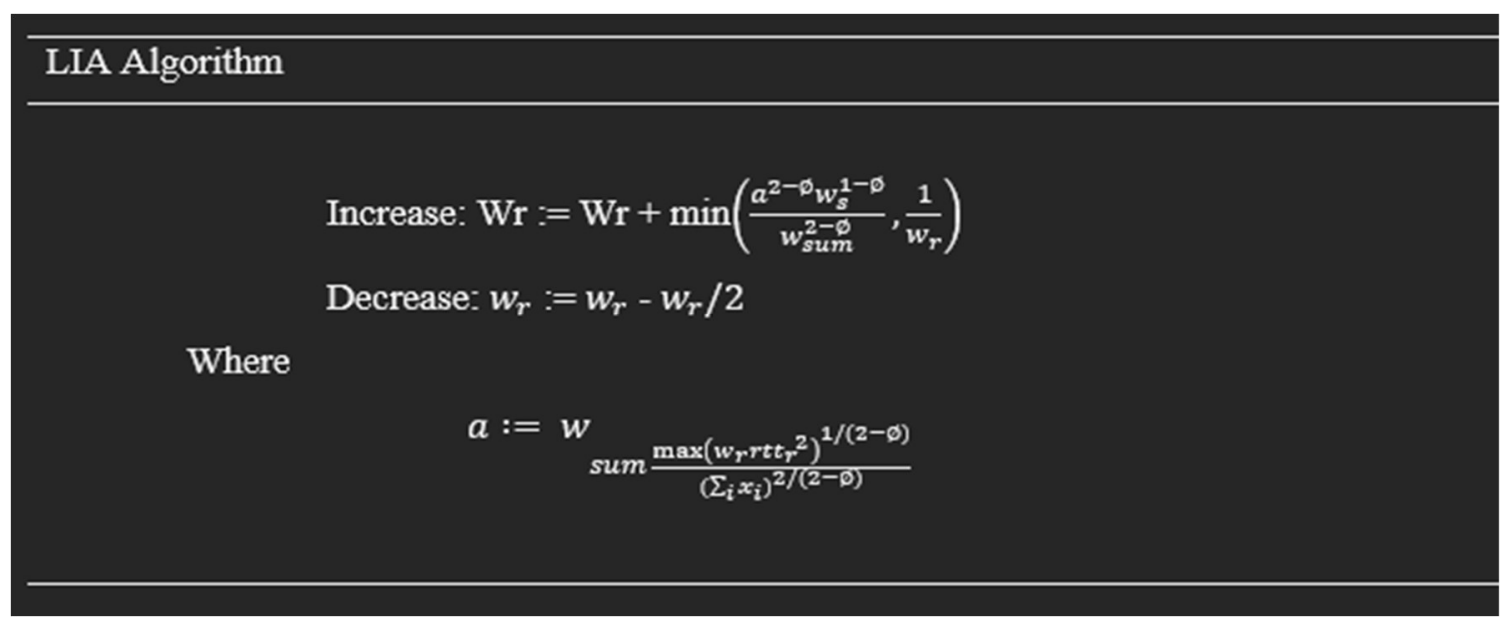

3) WVEGAS: WVEGAS is an extension of the TCP Vegas congestion control, WVEGAS congestion control has a weight regulating mechanism for each path that is separate from the cwnd increment mechanism. 
WVegas will perform the calculation between cwnd, average RTT and best RTT. If the calculation is not following the provisions, the cwnd value for each path will continue to increase, whereas if it is appropriate then the weight setting will be made based on the sending rate of each path [16].

\section{RESULTS AND DisCUSSION}

In this research, two types of tests were carried out to analyze the performance of congestion control LIA, CUBIC and WVEGAS. First, a QoS (Quality of Service) test is carried out using the Wireshark application to get objective results based on the real conditions of the current network then compare the results obtained to be assessed, which congestion control is better. Second, QoE (Quality of Experience) testing is carried out by giving a questionnaire to the respondent and then the respondent provides an assessment related to the video played by the examiner. The test is done by asking the respondent's opinion using a media questionnaire because to get QoE is to take data from the user experience, the parameters used to determine the quality of congestion control in terms of QoE is to see the quality of the video to be played based on parameters of image quality, sound quality and buffering time during video playback.

TABLE 1

CONFIGURATIONS OF EXPERIMENTS

\begin{tabular}{l|r|r}
\hline Environment & Packetloss & Traffic \\
\hline Env.1 & $0 \%$ & Yes \\
Env.2 & $1 \%$ & Yes \\
Env.3 & $0 \%$ & No \\
Env.4 & $1 \%$ & No \\
\hline
\end{tabular}

Table 1 describes the environment that will be used for testing. A $1 \mathrm{~GB}$ movie and a 51MB video clip were used as testing media. Multiple test environments will be created by varying packet loss and adding load to the network path for LIA, CUBIC and WVEGAS congestion control testing. The bandwidth used is 4Mbps with background traffic that matches the bandwidth size.

\section{A. QoS Testing Result}

In the QoS (Quality of Service) test, throughput is used as a parameter to measure the quality of the network during the video streaming. Testing is done using VLC as a medium for the client and server to stream video. Taking the tester throughput using the Wireshark. Testing is done 10 times for each environment. The test time to take throughput is 5 minutes for 1 video playback. 

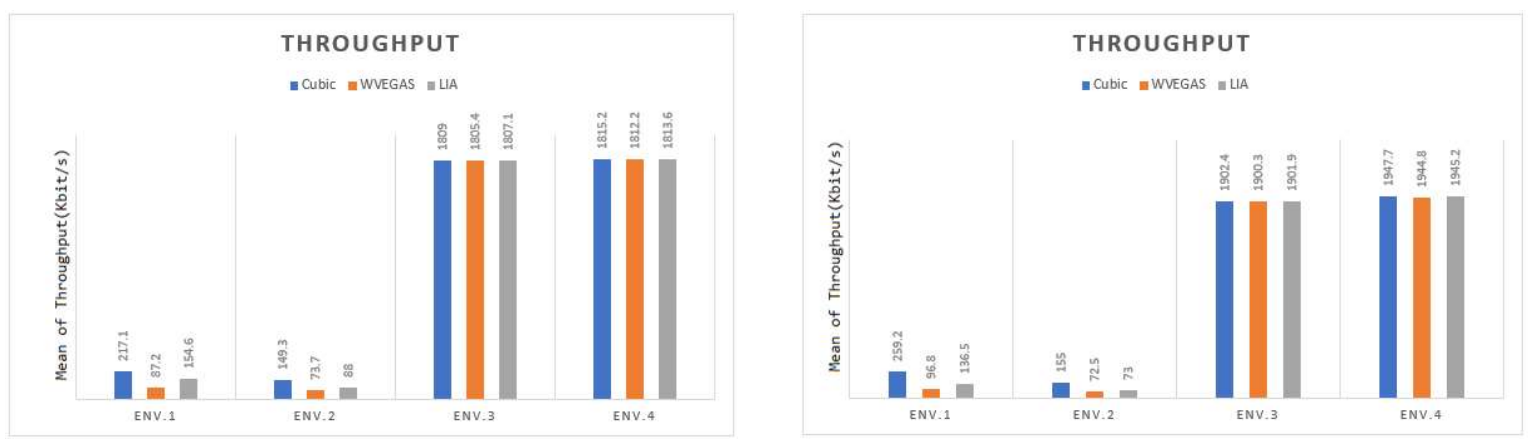

Figure 4. Throughput Results; (Left) Movie Streaming, (Right) Video Clip Streaming

Figure 4 shows the average throughput of the movie and video clip playback. There are differences in the results of each congestion control, environment and type of video played. In testing, packet loss and load are used on the network to test the performance of the congestion control. Based on the test results, it is proven that packet loss and network load affect throughput. Figure 3 shows that cubic congestion control has better performance than WVEGAS and LIA.

From the QoS (Quality of Service) test, it is known that the throughput value for environment 2 is the smallest. This occurs because the network environment 2 is experiencing $1 \%$ packet loss and has background traffic. In environment 1 , the throughput value is slightly better than the throughput than in environment 2 because there is no packet loss in environment 1 . In environment 3 , the throughput value is much better than in environment 1 and 2 because there is no packet loss or background traffic. And in environment 4 the throughput value obtained is slightly higher than in environment 3 because there is no background traffic, but $1 \%$ packet loss is given, where packet loss is a symptom of congestion which then causes active congestion control and increases throughput from normal network conditions (environment 3).

\section{B. QoE Testing Result}

In QoE (Quality of Experience) testing, image quality, sound, and buffering are used as parameters to measure the quality of streaming video. The test was carried out by displaying the results of the video streaming recording that had been prepared to 30 respondents. To get the respondent's results, we used a questionnaire indicating a quality scale. 1 is for the worst score and 5 for the best score. The video playing time for each environment is 5 minutes.
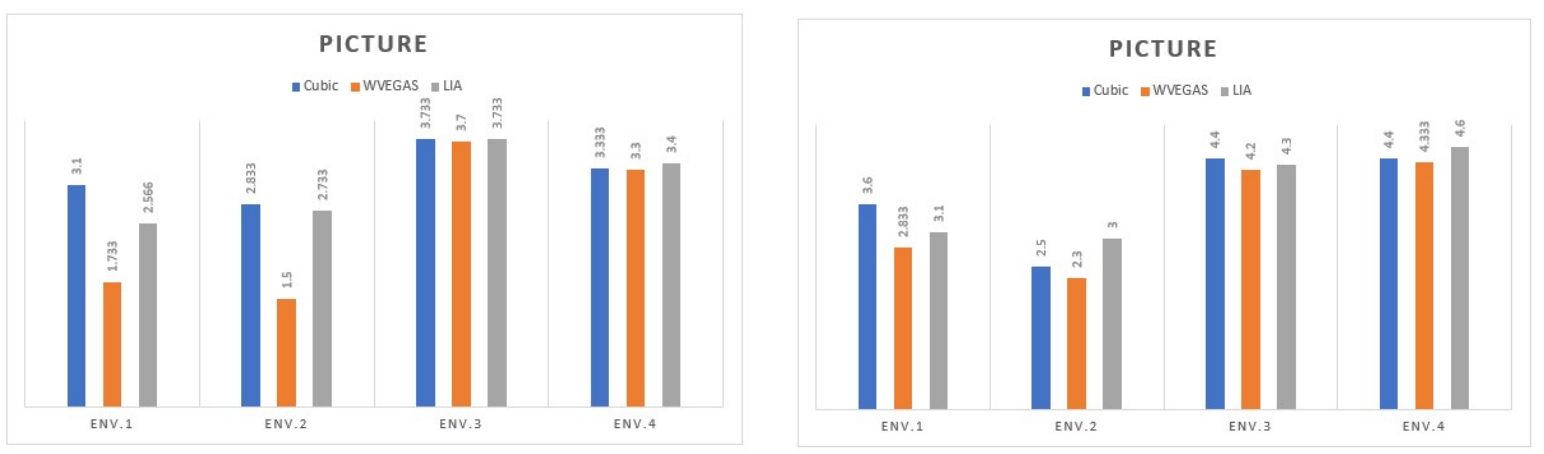

Figure 5. Picture Quality; (Left) Movie, (Right) Video Clip 
Figure 5 shows the QoE (Quality of Experience) test results on the image quality of the video displayed to the respondent. Based on the data we can find out the difference in image quality between congestion control, environment and what type of video is being played.
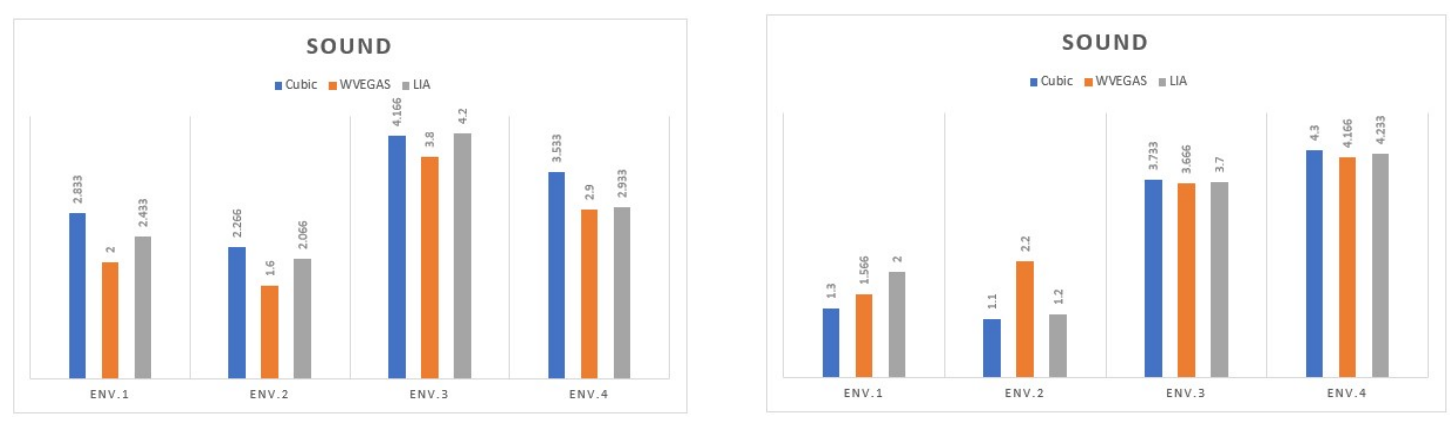

Figure 6. Sound Quality; (Left) Movie, (Right) Video Clip

Figure 6 shows the results of the QoE (Quality of Experience) test on the sound quality of the video displayed to respondents. The results show the difference in sound quality between congestion control, environment, and what type of video is being played.

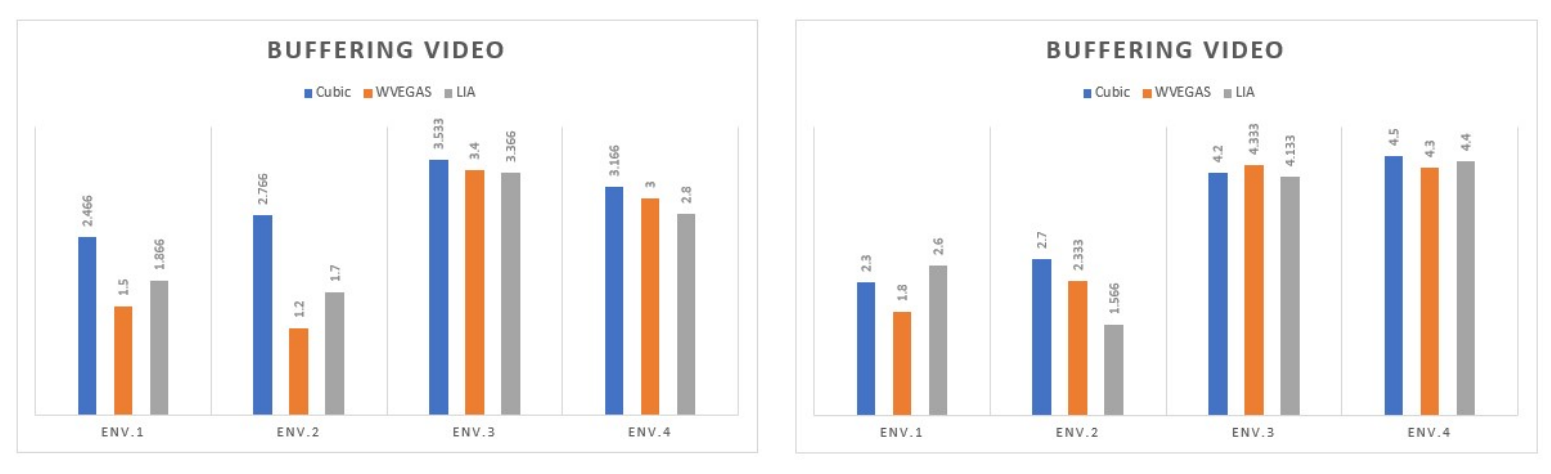

Figure 7. Buffering Video; (Left) Movie, (Right) Video Clip

Figure 7 shows the QoE (Quality of Experience) test results on the buffering quality of the video displayed to respondents. The results show the difference in buffering quality between congestion control, environment and what type of video is being played.

From the QoE (Quality of Experience) test, it appears that congestion control can affect the QoE assessment and has an attachment to the QoS value. Although sometimes the QoE value does not match the QoS value obtained from the test, the QoE test results still show that QoS can predict the results. The greater the difference in QoS values, the more visible the difference in video quality is displayed on QoE. The QoS results show that the cubic congestion control has the most optimal quality, as well as the QoE results although there is a slight difference because the assessment of each respondent is subjective.

From the results of the experiments conducted, the cubic congestion control algorithm has the best performance in terms of throughput, throughout the experimental environment. Meanwhile, in the assessment of image quality, the Cubic algorithm also has the best performance in environment 1, 2 and 3, while the LIA algorithm has the best quality in environment 4, although it is not that far off with the Cubic algorithm. 
This can also be seen in the sound quality assessment, where the Cubic algorithm is superior in 1, 2 and 4 environments while the LIA algorithm is superior in 3 environments. Finally, in the assessment of video buffering, the Cubic algorithm excels in experimental environments 1,2,3 and 4 in movies, and 2,3,4 in video clips.

\section{CONCLUSION}

From the tests that have been done, several conclusions have been drawn. First, multipath TCP and the type of congestion control affect the quality of QoE and QoS testing of streaming video. Second, the QoS value affects the quality of QoE. The QoE obtained has a relationship with QoS. The QoS value can be used to predict the QoE results. The greater the difference in the QoS value of each congestion control, the more visible the difference in video quality is displayed. And from the whole test, the Cubic algorithm shows its superiority in terms of performance in almost all experiments conducted.

\section{ACKNOWLEDGMENT}

Thank you, Telkom University, for being a forum for researchers to develop research in this journal. Hopefully, this research can contribute to technological advances, especially network technology.

\section{REFERENCES}

[1] M. Yasuomi and I. Yoshihiro, "Study on Effect of Congestion Control of Multiple TCP on Web-QoE," in IEEE Consumer Electronics (GCCE), 2015.

[2] C. Chia-Hu, L. Yu-Tzu and W. Ja-Ling, "Adaptive Video Learning by the Interactive e-Partner," in IEEE Digital Game and Intelligent Toy Enchanced Learning, 2010.

[3] G. Regel, P. Juan, E. Manuel and P. Carlos, "Hybrid Delay-Based Congestion Control for Multipath TCP," in Mediterranean Electrotechnical Conference(MELECON), 2016.

[4] B. Shadi, C. Cihat and S. Muge, "Evaluation of MPTCP Congestion Control for DASH," in Consumer Elecronics - Berlin (ICCE-Berlin), 2017.

[5] A. B. Letaifa, "SSIM and ML based QoE enhancement approach in SDN context," in Advances in Computers, Amsterdam, Netherlands: Elsevier, vol. 114, pp. 151-196, 2019.

[6] M. Jorge, G. Yuanzhi and G. Mario, "MPTCP Path Selection using CapProbe," in IEEE Wireless Communications and Networking Conference (WCNC), 2018.

[7] P. Anilal, B. V. Sainandan and S. S. S. Sai, "Experimentation and Analysis of Multipath TCP," in IEEE Advanced Networks and Telecommunications Systems (ANTS), 2015.

[8] P. M. Rahaman and C. Kang, "Improving WiFi Network Performance Under MPTCP User," in IEEE Computer Communication and Networks (ICCCN), 2017.

[9] H. Sangtae, R. Injong and X. Lixong, "CUBIC: A New TCP-Friendly High-Speed TCP Variant" in Operating Systems Review, 2008.

[10] L. Tuan-Anh, "Improving the Performance of Multipath Congestion Control over Wireless Networks," in Advanced Technologies for Communications (ATC 2013), 2013.

[11] C. Yu, X. Mingwewi and F. Xiaoming. "Delay-based Congestion Control for Multipath TCP," in Network Protocols (ICNP), 2012.

[12] M. Ryota, C. Dirceu, K. Kazumi, N. Daiki and I. Takeshi, "Multipath TCP Path Schedulers for Streaming Video," in IEEE Communications, Computers and Signal Processing (PACRIM), 2017.

[13] [5] D. Wischik, C. Raiciu, A. Greenhalgh and M. Handley, "Design, implementation and evaluation of congestion control for multipath TCP," in Proceedings of USENIX Conference on Networked Systems Design and Implementation (NSDI), 2011. 
[14] C. Volodymyr, R. Vasyl, B. Halyna and K. Andriy, "QoS/QoE correlation modified model for QoE evaluation on video service," in Modern Problems of Radio Engineering, Telecommunications and Computer Science (TCSET), 2016.

[15] N. Kien, J. Yusheng and Y. Shigeki, "Improving WiFi Networking with Concurrent Connections and Multipath TCP," in A World of Wireless, Mobile and Multimedia Networks, 2013.

[16] C. Chang-Yong and L. Zhouchen. "A Study of Video Buffering Mechanisms and Playing Speed Tuning Techniques," in IEEE International Conference on Computer and Communications (ICCC), 2017. 